\title{
Political influence of online platforms: YouTube's place in European politics
}

\author{
Yu. Y. Kolotaev ${ }^{1}$, K. Kollnig ${ }^{2}$ \\ ${ }^{1}$ St. Petersburg State University, \\ 7-9, Universitetskaya nab., St. Petersburg, 199034, Russian Federation \\ ${ }^{2}$ University of Oxford, \\ Oxford OX1 3QD, United Kingdom
}

For citation: Kolotaev Y., Kollnig K. Political influence of online platforms: YouTube's place in European politics. Vestnik of Saint Petersburg University. International Relations, 2021, vol. 14, issue 2, pp. 225-240. https://doi.org/10.21638/spbu06.2021.206

Social media platforms are today one of the primary means of political expression and campaigning. The growing entanglement of politics with the online sphere raises interest in how these new types of media shape the social and political reality. While previous research tends to focus on Twitter and Facebook, limited works exists on the political influence of YouTube. This is even though YouTube is the second-most visited website and provides unique ways of engaging users and disseminating political messages through the combination of rich communication functionality with immersive audio-visual media content. Unfortunately, the influence of social media platforms such as YouTube on politics is difficult to analyse, due to a lack of transparency and qualitative data. Independent researchers and the public have limited ways to access meaningful statistics about the video service. This article derives a taxonomy to classify YouTube's ways of political influence from a case-study-driven analysis of YouTube in the European political landscape. The authors' taxonomy aligns with traditional theoretical concepts from the media effects theory, particularly framing, priming and agenda-setting. A brief discussion of the current regulatory landscape in the EU is provided, and gaps are highlighted that might need improvement. The authors conclude by considering the question of whether YouTube is a political actor.

Keywords: YouTube, social media, online platform, European Union, content moderation, political content.

\section{Introduction}

There are so many major moments that lead up to Election Day: debates, caucuses, primaries. But the moments that matter most [...] happen quietly and quickly in micro-moments, when undecided voters become decided voters, often by going online.

Google (2016): "How Political Ads and Video Content Influence Voter Opinion"

Contemporary politics relies increasingly on online and social media communication. The growing entanglement of politics, whether global or local, with the online sphere raises interest in how these new types of media shape the social and political reality. Generally, the question of what role media play in politics has long raised concerns and inter-

(C) St. Petersburg State University, 2021 
est in the $20^{\text {th }}$ century. Yet, the shifting nature of media from traditional forms (mass media) to the social network type (social media) reveals new patterns of the media's role in politics.

There is a great number of the new-type media that emerged over the last two decades. Commonly, social networks, such as Facebook and Twitter, attract most attention in this regard. However, there is one medium of even higher relevance, that has still received less attention, despite it being the most widely circulated medium around the world: YouTube. It is the world's most famous video content distributor, started as a service to distribute videos in 2005. It was quickly acquired by Google the year after and today offers sophisticated functionality for people to connect and interact, thereby shifting from sole video distribution towards a social media platform.

While YouTube typically refers to itself as content 'distributor', there is growing doubt about its neutral role. The Cambridge Analytica scandal demonstrated how the masscollection of personal data might be used to sway the opinions of the electorate through personalised advertising. Since personalised advertising is the core of YouTube's business model, it has been argued that the service must drive user engagement to make a profit, regardless of the consequences for society [1]. Many scholars hypothesise that the content selection of YouTube radicalises online discourse and thereby threatens the political debate, democratic culture, and societal cohesion [1-3]. Conclusive evidence remains widely absent because of the difficulty in conducting meaningful analyses.

A major challenge in analysing the impact of YouTube is the lack of transparency and qualitative data. Independent researchers and the public have limited ways to access meaningful statistics about the video service, scrutinise its practices, and hold it to account. YouTube's influence on politics and society remains little understood.

To address this gap, we conduct a case-study-driven analysis of YouTube's political influence. Our analysis will focus on European politics and the way YouTube is included in political and social interactions as a medium and how this might render YouTube a political actor. Europe, and the European Union in particular, provide a good example for a multilevel study of YouTube's interactions and activities on the national and supranational levels and its compliance with regional digital legislation. For more coherence, we first categorise the different dimensions in which YouTube may exert political influence and substantiate these observations with examples. In doing so, we will apply concepts from media analysis, particularly framing, priming and agenda-setting. Then, we relate our findings to the current EU framework, and identify potential gaps that might need regulatory attention.

\section{Background}

Since its creation in 2005, YouTube has been a video-hosting for user-generated content. Initially, user-generated videos were perceived as a poor basis for stable income and profit [4]. But already in 2007, some observers have noted not only the success of the business model but also the increasing influence of YouTube, calling the coming 2008 US Election "the YouTube election" [5]. There increasingly emerged a self-identification dilemma for YouTube: while being an open video platform for everyone, it also had commercial interests and started to enter the political arena. Politicians began to use YouTube as an instrument to access a wider public audience. This tendency of the platform's "politization" continued during the EU elections in 2009, when YouTube launched an EU elections channel under the name "Questions for Europe" [6]. 
In the early 2010s, the first academic articles appeared related to the political dimension of YouTube [7]. Considering the term "platform" as a framework for YouTube's selfidentification, some researchers highlighted that the video-content provider becomes one of "the curators of public discourse" [7]. Following up, in 2014, L. Ricke published a book on the impact of YouTube on U.S. Politics [8]. Motivated by new insights into the ways the platform influences social relations, he emphasised that "although primarily situated as a commercial enterprise, YouTube has become a pervasive and vital political communication tool" [8, p. 1].

Indeed, in the same year, YouTube publicly declared itself an "important platform where citizens engage with their governments and elected officials" (emphasis added), thereby showing that the platform has become an essential tool for political communication [9]. This self-manifestation as a "platform" signifies the most crucial theoretical questions of whether YouTube acts as an open, engaging and equally accessible "forum" for political communication (without any kinds of manipulation or distortion), or increasingly as a political actor.

The openness of YouTube raised doubts very early on. T. Gillespie noted in 2010 that "to the degree that information intermediaries like YouTube claim to be open... the kinds of interventions and choices these providers actually do make can be harder to see" [7, p. 359]. Indeed, the term "platform" is a discursive trap. It suggests openness and neutrality, which can never exist if content is filtered and ranked, as done in the case of YouTube. In 2015, a paper on online campaigning analysed how "traditional and non-traditional" actors can spread their messages on YouTube, underlining how YouTube is skewed towards the traditional mass media outlets and struggles to democratise the public debate truly [10]. Indeed, the usage of the term "platform" to characterise YouTube indicates that there is a lack of "sufficiently precise language for attending to these kinds of interventions and their consequences" [7, p. 360] making the idea of YouTube as a platform bounded, if not distracting from the core issues.

Recent studies on social media also reveal "that alongside immediate communication power (such as networking, networked and network-making power), social media also has ... 'systemic opinion power"' [11, p. 846]. Such power means the ability to "create dependences and influence other players in a democracy" [11, p. 846]. In the case of YouTube, this might lead to the creation of a distorted picture of reality [1], by changing, shifting, and radicalising the public discourse on political matters.

Besides this, another question remains understudied. Insufficient attention is being paid "to the growing trend by... YouTube to move into the active role of content editors" [11, p. 849]. The matters of content moderation become yet another prerequisite to outline the actorness of YouTube. To some extent, the possibility to frame and limit inappropriate political content has been manifested in the 2020 guidelines on YouTube's support for elections [12]. This, together with the aforementioned incidents of access limitations, has already amplified the concerns of the possible "quasi-governmental" actorness of YouTube [13].

Overall, there emerge two significant issues related to YouTube. First, YouTube possibly transcended its self-image of a "platform", having already become a quasi-actor in politics. Second, the growing systemic opinion power of platforms - such as YouTube might require new remedies, given the impact on freedom of speech and equal participation in the democratic process. 


\section{Power and actorness of mass and social media}

Both identified issues relating to YouTube raise the question of what political power a platform or media can pose. This question has a long history in political and media studies, even before media based on an online network logic, i. e. social media, started to challenge traditional mass media.

In the early 2000s, political and media studies came to several basic assumptions about the political and discursive powers of media, including the abilities of framing, priming and agenda-setting [14-17]. The three concepts are highly interrelated. Framing is a "process of culling a few elements of perceived reality and assembling a narrative that highlights connections among them to promote a particular interpretation". Priming and agenda-setting supplement this process by raising "the salience or apparent importance of certain ideas" and "defining problems worthy of public and government attention" respectively [18, p. 164]. Through a set of discursive practices, the context of a certain message, event or statement can be changed by the media in a certain way corresponding to a particular agenda. This highlights the potential threat of systemic opinion power.

These abilities of (traditional) media were detected in the context of the mass media influence on public and political discourse. In terms of critical discourse analysis, interconnections of politics and media resulted in the emergence of mediatised politics which "is sometimes seen as the colonization of politics by the media, and sometimes seen as the colonization of the media by politics" [19, p. 200]. This premise leads to the understanding that in the contemporary context as well as previously "restricting the role of the media to their primary function of information dissemination is not only a deficient description of the media, but also means distorting and underestimating the legitimate active role of the media in the process of opinion formation" [20, p. 183].

Even though the medias' discursive influence has been recognised, a relevant question remains. To what extent are the features of traditional mass media relevant for social media? Initially, the term "social media" itself was contested ("because it implies that traditional media do not encompass a social dimension") [21, p.23]. The interactive and networking possibilities of social media platforms showed a shifting approach to media. As highlighted by U. Klinger and J.Svensson, "network media logic differs from traditional mass media logic in three different but interrelated dimensions: the production of media content, the distribution patterns of information, and the way people use media" [21, p. 34].

As for the political sphere, the shift from mass media logic to social media has shown limited impact on how political actors use media. Even in contemporary social relations, the broadcasting function (and not the networking possibility) "continues to be central to political social media practices" [21, p. 24]. The interactive potential of social media is used only partly $[22 ; 23]$. This shows continuity in the existing practices of media usage by political actors. Even if broadcasting remains the essential function of media, the network logic of social media has altered and changed the way politically relevant information is disseminated and received by the social media users. Already in 2011, Foreign Affairs asserted "the political power of social media" [24], showing that social media platforms are increasingly becoming active participants and mediators in political (and international) relations. This political power can be utilised by social media and other stakeholders in different ways (including framing, priming or agenda-setting). However, there is no strict 
answer to the question of whether the existence of such power shows the presence of actorness.

Answering this question, another researcher on media actorness, B. I. Page, stressed, that the "political actor" concept (applied to the media or anyone else) "implies observable action that is purposive (though perhaps functional rather than consciously intended) and sufficiently unified so that it makes sense to speak of a single actor" [25, p. 20]. In this context, there is a need to examine the potential purposive and unified actions of a social media, i.e. YouTube, to understand its possibility to be perceived as a political actor. In doing so, we will assess potential ways, in which YouTube can perform political actions or display political power and to what purpose. To complement this assessment, we will examine the possibility to apply the concepts of framing, priming and agenda-setting to the actions of the social media.

\section{Types of political influences}

YouTube's prominence in online media gives rise to political influence in many ways. Motivated by how traditional mass media spreads certain ideas or beliefs, we propose that these ways of influence fall in the case of social media such as YouTube into three distinct categories: direct (framing effect), indirect (priming effect), and hybrid (agenda-setting effect) ways. The following discusses how each of these three groups act politically, by drawing on relevant examples from Europe. While our choice of examples is geographically restricted, similar ones might be found in other regions.

In our case study, we drew on diverse examples from European politics connected to YouTube's activity beyond its status as a solely economic entity. The selection of the examples was based on a qualitative analysis of primary and secondary sources, including official statements by public and private bodies, as well as newspaper articles. The presented taxonomy of the types of political influences arose from a systematic comparison and thematic analysis of the identified sources. We summarise the results of our case study in Table 1.

Table 1. Summary of case-study in this document: The ways of YouTube's political influence fall into three distinct categories that align with three major media effects

\begin{tabular}{|l|l|l|}
\hline \multicolumn{1}{|c|}{ Direct (framing) } & \multicolumn{1}{|c|}{ Indirect (priming) } & \multicolumn{1}{c|}{ Hybrid (agenda-setting) } \\
\hline Lobbying & (Personalised) Advertising & $\begin{array}{l}\text { Content Moderation and } \\
\text { Censorship }\end{array}$ \\
\hline Direct Messaging & $\begin{array}{l}\text { Hyper-Personalisation } \\
\text { and Platform Design }\end{array}$ & Hosting of (Political) Content \\
\hline Self-Marketing & & \\
\hline
\end{tabular}

\section{Direct}

Direct political influence can be categorised into lobbying, direct massaging and selfmarketing, depending on the audience and the tools used to promote a particular idea or interest of YouTube. All of these categories imply to a significant degree the framing effect 
due to the inherent logic of the promoted message, and allow YouTube to act as a political agent, assembling a narrative with a particular interpretation in a unified and purposive way. Still, the framing happens not through the dissemination of a textual interpretation of particular events while highlighting news, but through the promotion of a causal interpretation or moral evaluation of certain problems [26].

\section{Lobbying}

Corporations have long used lobbying to influence policy in their favour, and YouTube is no exception. Ample theoretical evidence proves that lobbying can be effective. Famously, Mançur Olson argued in his 1965 The Logic of Collective Action that small groups can often override the interests of large groups - such as the unorganised societal majority - due to the different organisational costs [27]. More recent economic research provides empirical evidence that indeed "economic elites and organised groups representing business interests have substantial independent impacts on U.S. government policy" [28, p.565]. The average YouTube user might be at a disadvantage to the shareholders behind the company.

In the EU, in-house lobbying becomes a way to influence certain relevant decisions on a multi-level basis. A recent attempt in this regard is the debate around the EU Copyright Directive. YouTube CEO Susan Wojcicki directly stressed in an open letter on YouTube's 2019 achievements, that she was involved in a dialogue with policymakers. On the local level, it included the explanation of the creator economy's value while "meeting with governments and inviting local creators to help explain to legislators how Article 17 (formerly known as Article 13) could affect creators" [29]. The attempt to "advocate for responsible copyright reform" becomes a frame used by YouTube while lobbying. Still, the framing of the debate on EU Copyright Directive shows YouTube's self-interest, which gives the lobbying a purposive nature. The self-interest is connected to the increased liability of the platform under the Directive. Yet, direct lobbying was not the only instrument to frame the public opinion on Article 17.

\section{Direct messaging}

Another way of YouTube's political influence is the engagement of its own users in the political process through direct messaging. This approach is similar to lobbying, but has a different audience. While the primary target in lobbying are policy makers, direct messaging aims at individual users to sway their opinion in one way or another. Due to the large YouTube user base of more than 2bn monthly active users [3], YouTube is in a special - if not unique - position to be able to deliver (political) messages to such a vast number of users. It is difficult to perceive how any traditional news company or communication medium could match the reach of YouTube.

In the similar case of the EU Copyright Directive, YouTube tended to frame the users' and creators' opinion to strengthen its lobbying capabilities. In a clear statement on the negative implications of the Directive Susan Wojcicki encouraged content creators to "tell the world through social media (\#SaveYourInternet)... why the creator economy is important" [30]. In its appeal to "take action immediately" YouTube started, as characterised by several media, "one of its most aggressive consumer-facing lobbying efforts" 
[31] to protect the platform's profitability [32]. In the end, dozens of YouTubers from EU countries supported the \#SaveYourInternet campaign leading to the improvement of the Directive's final version in 2019.

To our best knowledge, there are no apparent examples of YouTube using direct messaging to promote the political interests of other corporations. However, YouTube engages in various collaborations to produce video content, through which the platform necessarily promotes a certain agenda. YouTube might also promote the political interests of other organisations indirectly (e. g. through the dissemination of advertising), as we discuss in the following sections.

\section{Self-marketing}

Self-marketing is another instrument through which YouTube acts as a political agent. The company uses public communications, including its blog, policies and website, to frame how it is perceived. One of the most well-known examples is the self-characterisation as a "platform", which YouTube has long used in public communications [4]. The term suggests a neutral role as a content distributor, and indeed, YouTube currently faces limited liability for content on its platform under EU and US law because of this role. Yet, platforms, such as YouTube, choose what content is seen by its users and distribute content for a profit. The self-framing as a neutral platform distracts from this role, and does not allow for the precision needed to scrutinise the business practices of YouTube [4] and its political influence.

During the last years, contradictory examples of YouTube's self-marketing can be found on the blog of the company [33]. Here, YouTube presents itself as an effective - if not essential - way to reach voters in elections. Thereby, the platform puts forward a narrative to legitimise new ways of influencing public opinion, notably personalised ads. At the same time, more recently, YouTube tried to stress its efforts to become a "reliable source for news and information" and an "open platform for healthy political discourse" [12]. This dichotomy reveals the inherent contradiction in YouTubes role as a profit-oriented company and as a political actor responsible for equal access to an open social dialogue on the platform.

\section{Indirect}

There is a range of indirect ways in which YouTube may distort the political agenda. The notion of indirect influence includes priming which occurs by emphasising certain politically and socially relevant ideas and stressing those arguments that can sway the user's opinion. An example of indirect influence are ad campaigns that "do not promote a certain party or candidate, but the subject matter and message would favour certain candidates and parties because of their aligned agenda" [34, p. 11]. This brings indirect influence close to agenda-setting, but, in its nature, it is built on indirect stimulus. Thereby, one of the most important aspects of priming is its dependence on the idea's accessibility in the human mind based on the frequency and recency of the user's exposure to an idea [16]. A platform can manipulate both the frequency and recency with which content appears in the user newsfeed or recommendations. The main instruments are the advertising and personalisation tools ingrained in the design of YouTube. 


\section{(Personalised) Advertising}

YouTube acts not only as a platform for videos, but also for ads. Just as for sharing videos, almost everyone can deliver political messages on YouTube, by purchasing advertising on the platform, given they follow the rules and guidelines set by YouTube. By allowing for advertising (whether political or not), YouTube determines what opinions users are exposed to, and thereby influences public discourse. A similar line of arguments applies, as laid out in the rest of this article. While YouTube commits itself to quality checks on the ads with special rules for political ads, YouTube's moderation of ads poses the same tensions around freedom of speech, commercial interests, and the health of public discourse as the moderation of video content. YouTube's advertising moderation (alongside content moderation) constitutes a de facto monopoly in conditioning the distribution of video content on the Internet. It derives from YouTubes market dominance in video hosting and the absence of transparency measures in the moderation process (for example, the decision processes to determine the rules for political ads are not published).

This problem is also present in the European context. Isabella Adinolfi MEP raised the concern that "some YouTube contributors are claiming that their videos have been unfairly deprived of the advertising proceeds from the platform" [35] with the EU Commission. This stresses the potential negative outcomes of YouTube's advertising moderation. Still, the European Commission notes that social media platforms "remain free to organise matters such as advertising policies" [36]. Thus, even under the threat of violating several fundamental rights, the advertising rules of platforms such as YouTube in the EU remain subject to limited judicial safeguards.

Through its ability to impact and define the number and nature of ads, YouTube can raise the salience of certain messages and ideas. Even if not used directly or deliberately, it could "in practice constitute a violation of the freedom of expression" [35] or lead to an intentional or unintentional priming effect. Both consequences originate not only from YouTube's "advertising power" itself but also from the platform design. For example, algorithmic (advertising) moderation - as done on YouTube - is known to struggle to understand the true meaning of content, given the current limitations of the underlying natural-language processing technology (NLP).

\section{Hyper-personalisation and platform design}

YouTube - as other social media - provides a hyper-personalised user experience. This hyper-personalisation of YouTube is expressed in many ways, including video recommendations on the landing page and the next-up recommendations, the search functionality, and the obligation to create a user account to access certain functionality of YouTube. This raises concerns about the design goals of YouTube.

There are doubts about how well the design of YouTube is aligned with the interests of its users. More time spent on YouTube means more opportunities for Google to serve ads, and potentially increase revenue. Because of this, the service has widely been accused of luring users into spending ever more time on the platform. For users, more time on YouTube might bear negative effects on their well-being (including different kinds of social and psychological harms). An emerging body of literature on digital self-control 
suggests that users often struggle to exert a meaningful choice over their technology use, and that platforms - such as YouTube - apply patterns to exploit human psychology for their ends [5].

The way, in which YouTube is designed, matters, and it can have negative effects on individuals, society and the public discourse. The personalisation of content gives the platform a clear picture of a user's preferences and information consumption. Provided a malicious intent, such knowledge can give broad opportunities for intentional priming. The personalisation mechanism might have the capacity to manage the amount and recency of certain content, which can be further used to create an indirect stimulus for certain biases in social and political preferences.

Even if the malicious use of personalisation is an unlikely (but daunting) prospect, two pressing issues still arise globally. First, YouTube's algorithms might radicalise people and their opinion due to the algorithm's potential bias toward inflammatory content (as it tempts users to stay longer on the platform) $[1 ; 3 ; 37]$. It has been argued that "YouTube monetizes influence for everyone, regardless of how harmful their belief systems are" [38, p.43]. This can be an example of "unintentional" priming through constant suggestions of watch time maximising content. The second issue is connected to the recommendation algorithm itself. Due to its proprietary nature, the underlying incentive structures remain opaque [39]. Given the priming possibilities, there is a clear need for increased algorithmic transparency.

\section{Hybrid}

There are further ways of YouTube's influence on elections or political developments that classify neither as direct nor indirect ways. Rather, such influence has a hybrid pattern, wherein the platform utilised its intermediary position to define the type of content that is visible for users, thus emphasising certain issues over others. This suggests that YouTube possesses an agenda-setting capability, which appears in two forms. First, there is the moderation and censorship of content, i. e. the ability to define what and how content is (or is not) shown on the platform. The second form is the hosting of political content itself. Being an intermediary in this context includes the obligations for fair representation of the political debate. The exclusion or inclusion from the platform might be of crucial importance for the political representation and power balance. Being able to impact the political landscape gives YouTube unique levers of influence in politics.

\section{Content moderation and censorship}

According to YouTube's self-description, its global aim is "to give everyone a voice and to show them the world" [40]. This hints towards the fundamental problem of ensuring proportionality in free speech and deciding what world users should see on the platform. One of the most pressing problems related to free speech is currently the dissemination of different kinds of false information, fake news and hate speech in the digital environment. Ample empirical research suggests that false information spreads more easily on social networks because of its higher information content compared to truthful information [6]. This raises several crucial issues around YouTube. How should YouTube act on disinformation and misinformation? Should it be removed, or rather 
its spread be reduced? These questions become even more difficult as different national contexts might lead to different conclusions, yet YouTube operates as a global company.

The scale of harmful content on YouTube has brought the platform in a position, where it needs to determine and regulate the (political) agenda of the platform. To fulfil this role, YouTube has been assigning ever more human moderators to content moderation, and been improving on their algorithmic content screening through natural language processing and machine learning [41]. Despite these efforts, problems with "over blocking" (wrongly taking down content, such as satire, remixed, and whistle-blowing content), the balancing between freedom of speech and "freedom of reach" [42] (reducing the visibility of problematic content, instead of removal), and lack of accountability and transparency persist in content moderation. External instruments to balance YouTube's power over content remain widely absent, sparking concerns that the current self-censorship of YouTube might do more harm than good [42].

The way in which YouTube performs the moderation can differ depending on the region. For example, fact-checking features for video searches are offered only in certain countries of Europe (and the US) [43]. This leads to disproportionate attention of YouTube to certain countries, while missing others, and does not live up to the global nature of YouTube's role in the information space.

Beyond that, there are some cases that expose the challenging nature of YouTube fulfilling the role of a censor on its platform. One case comes from European politics. A French blogger accused YouTube of trying to censor an interview with the ex-President of the European Commission Jean-Claude Juncker [44]. Allegedly, YouTube threatened her further career prospects on the platform if she asked tough questions. Clearly, the extent to which YouTube could exert pressure on the blogger remains open. Yet, it reveals the potential pressure YouTube can exert on content creators and the political agenda.

An ongoing case is the complaint of the Russian government regarding the blocking of several news agencies by YouTube under the pretext of the violation of hosting rules. In a statement of the Ministry of Foreign Affairs of the Russian Federation YouTube's actions were considered as an "act of discrimination against Russian-language media resources" and "arbitrary censorship of content" [45]. The conflict revolves around diverging interpretations of free speech and harmful content, having led to widespread outrage of Russian officials and a draft bill targeting YouTube. This example demonstrates the increasing emergence of confrontations between the platform and a state, especially since state and media are often interrelated. Such kind of confrontation has a political nature and stresses once again several aspects of YouTube's actorness.

While determining the presence of a media or news agency and exerting thereby the agenda-setting function, YouTube acts in a functional (or purposive) way. The functionality is defined by the community guidelines and platform rules, which make its actions also unified.

\section{Hosting of (political) content}

Lastly, YouTube exerts political influence by hosting videos with political messages on its platform [46; 47]. Sometimes, these political messages might be obvious (e.g. in videos by politicians), sometimes this might be more subtle (e.g. by video producers conforming with certain political ideologies). Most of the content on YouTube is produced without 
the active oversight of YouTube. However, the design and rules of YouTube, including its monetisation model, sets certain incentives for the available content on YouTube. For instance, such might favour sensationalism (driving time spent on YouTube) over journalistic quality (potentially less sensational). Permitting certain kinds of content on its platform (as discussed in the previous point) can be seen as a direct way of YouTube's engagement in the political agenda on its platform.

Recent examples from across Europe suggest that YouTube increasingly becomes an important tool in political campaigning. Some of this is connected to the usage of YouTube as the dominant communication instrument. For instance, the current Ukrainian president Volodymyr Zelensky conducted his successful 2019 campaign for office almost entirely on YouTube. Other examples include the above-mentioned interview with JeanClaude Juncker and an interview of Emmanuel Macron with a young YouTuber ahead of the 2019 EU elections.

One of the most significant examples of YouTube's political influence is the so-called Rezo-case, wherein a German YouTuber produced a video ("Destruction of the CDU") in the run-up to the 2019 European Parliament elections. His video heavily criticised the leading German parties, particularly the CDU, the largest German party. By the election day, the video had reached more than 10 million views and sparked an unprecedented national discussion about the influence of online media on German politics. Ultimately, the European elections turned out as a disaster for the governing three-party coalition, reaching only $45 \%$ of the total votes, and only $20 \%$ amongst voters aged 18-24 [47].

The connection between the results of the elections in Germany and Rezo's video are still disputed, but this example revealed the potential audience reach and agenda-setting capability of the platform in a political context. Even if the video itself did not become a central factor in the voters' decisions, it shifted the public discourse in a politically highly intense pre-election period. Even if YouTube is only an intermediary in this situation, it possesses a certain actorness due to enable YouTubers to disseminate politically relevant information outside of traditional media.

\section{YouTube, a political actor?}

All the above-discussed kinds of political influence reveal a complex conflict in the perception of YouTube from a political perspective. The tension between YouTube as a political platform, that is to say a tool in political communication, conflicts with the recurring display of YouTube's actorness. To understand this conflict, it is essential to reconsider the features of an actor's behaviour, i.e. a unified and purposive nature of its actions.

Diverse examples of direct influence reveal YouTube's ability to manifest itself as a single actor pursuing its interest, e. g. in the context of European copyright regulations. While capable of pursuing political interests in a unified way, YouTube cannot be considered being as unified as traditional mass media. The main reason is the diversity of the content creators on YouTube, and the wide range of opinions and interests.

The purposiveness of YouTube's actions can neither give a straight answer to the actorness dilemma. Intentions and interests of YouTube are driven by financial motives. These aim at increasing advertising revenue rather than political power. The significance of financial incentives forces YouTube to stay apolitical. However, while "attempting to stay apolitical, YouTube consistently makes the political choice not to care about or pro- 
tect vulnerable communities" [48]. Exploitation of the political landscape is a central externality of YouTube's business model.

Those two aspects reveal that the actorness dilemma cannot be unequivocally resolved. To solve this dilemma, YouTube needs to be rebalanced towards the company's self-understanding around promoting free speech in the digital age. A first step could be the acceptance of political responsibility. As previously done by traditional media, being commonly referred to as the Fourth Estate, YouTube and other social media should acknowledge their role in the political landscape. This is even more important than in the case of traditional media due to the sheer size of the audience and the resulting power. A second option could be the depoliticisation of the platform. There is some evidence that the users do not always welcome the political role of the platform [46]. Still, considering the commercial interests of YouTube, this would lead to a decrease in profit and, thus, it is less likely to be realised.

A more realistic way to tackle the emerging political actorness of YouTube would be regulatory remedies on the national or supranational level, such as the European Union. These remedies may be particularly important since there lacks clarity as to how YouTube localises its standards and algorithms to a particular country, given the opacity of the algorithmic system.

\section{EU legislation}

The most realistic way to govern the political influence by YouTube are regulatory remedies imposed by a national or supranational authority. With the EU being one of the largest and most active regulators of digital technologies and social media, this section considers how the political power of the platform could be governed within the existing legislative framework of the EU. We summarise our analysis of the EU regulatory landscape in Table 2.

Table 2. Summary of our analysis of the EU regulatory landscape: Existing legislative framework in the EU and limitations concerning the regulation of the political power of YouTube

\begin{tabular}{|l|l|l|}
\hline Legislative framework & \multicolumn{1}{|c|}{ Foundation } & \multicolumn{1}{|c|}{ Problem } \\
\hline Media law & $\begin{array}{l}\text { Article 151 TEFU on the protection of } \\
\text { cultural diversity. }\end{array}$ & $\begin{array}{l}\text { Most media law regarding social stems } \\
\text { from EU member states. }\end{array}$ \\
\hline Data protection law & $\begin{array}{l}\text { Article 16 TFEU on the protection of } \\
\text { personal data. General Data Protec- } \\
\text { tion Regulation (GDPR) is main piece } \\
\text { of legislation. }\end{array}$ & $\begin{array}{l}\text { Limited enforcement and strong focus } \\
\text { on individuals, less on media. }\end{array}$ \\
\hline Competition law & $\begin{array}{l}\text { Articles 101 and 102 TFEU ensuring } \\
\text { fair competition. }\end{array}$ & $\begin{array}{l}\text { Current competition practices put li- } \\
\text { mited focus on data-related issues. }\end{array}$ \\
\hline
\end{tabular}

One of the most obvious approaches to tackle the power of YouTube might be to tighten EU media law, as suggested by Natali Helberger [11]. While the European Union was founded as a foremost economic project, it has over time developed beyond that, towards an emphasis of media plurality as one of its pillars. This is enshrined in Art. 151 TEFU (Treaty on the Functioning of the European Union), protecting cultural diversity in the 
EU. However, most media regulation remains in the hands of the EU member states, not the EU. Examples are the NetzDG and the Medienstaatsvertrag in Germany, proposing methods to decrease harmful content on online platforms while increasing proportionality of opinions. The current fragmented approach might not be enough, and media law needs to be evolved into targeting digital services.

Fragmentation around the regulation of data amongst the EU member states led to the introduction of the EU General Data Protection Regulation (GDPR) in 2018. The law combines tight rules on the use of personal data, i. e. data relating to individuals, with high fines of up to $4 \%$ of global annual turnover. YouTube's hyper-personalisation, particularly of advertising, would not be possible without the use of personal data. Despite the strict rules, enforcement remains the bottleneck of the legislation [49]. Data protection regulators with limited funding are tasked with taking on multi-billion-dollar companies. Indeed, problems around data are often not only issues of individuals - as governed by the GDPR - but problems for society at large. The GDPR was not designed to find solutions to these bigger questions, and the regulation of YouTube's political power might not lie within the realm of data protection regulators.

The area of competition law has been struggling with similar problems around enforcement. Corporate power and the abuse thereof are governed by Articles 101 and 102 TFEU. Competition authorities are tasked with enforcing these provisions, including overseeing mergers and acquisitions (M\&A). Despite this, Google has been able to expand its access to data and influence over the digital ecosystem by hundreds of M\&A transactions, none of which have been stopped $[49 ; 50]$. Arguably, the acquisition of YouTube itself in 2007 was the first of such "data mergers", followed by Admob, DoubleClick, Crashlytics, and hundreds of other companies. Despite this increasing influence over data and the digital ecosystem, competition authorities have argued that the increased access to data does not pose a threat to competition, and that M\&A transactions can go ahead.

To date, no designated EU regulation for social media companies, such as YouTube, exists. Instead, there are many different pieces of legislation, none of which aims at the political power of YouTube. Regulatory authorities do currently not have effective means to counterbalance the ongoing concentration of political power in the hands of YouTube. Despite this, there is a strong obligation for the European Union to protect equality in the democratic process: "In all its activities, the Union shall observe the principle of the equality of its citizens." (Article 9 Treaty on European Union.) This equality might be at stake. The European Union has realised the need to rebalance the digital ecosystem, emphasised by its Digital Single Market Strategy since 2015 and the upcoming EU Digital Services and Markets Acts. The pressure is high to find and implement appropriate remedies soon.

\section{Conclusions}

The present article has analysed the manifold ways, in which YouTube influences the political agenda. The platform does so in 1) direct ways, through communicating its views to policy makers, users and the general public, 2) indirect ways, through strong personalisation of the user interface, particularly personalised advertising, and 3) hybrid ways through the nature of YouTube itself as a video sharing platform that is increasingly used for political expression, with both positive and negatives impacts on the democratic society. 
Because of these various ways of political influence, YouTube acts sometimes as a political actor. While YouTube may not aim to change the political agenda, its current design often inadvertently does. This can put great power into the hands of a small, unelected group of circles behind the company. Current EU legislation struggles to provide effective remedies against this increasing political power of YouTube. The UK Competition and Markets Authority suggested putting the oversight of social media into the hands of a super regulator, which might be a role model for the EU [51]. The strong alignment of YouTube with financial incentives underlines the potential effectiveness of regulation: if the incentives are shaped adequately, YouTube will adapt. In the meantime, there will be limited need for YouTube to rethink its business model.

This article has shown that much of the power imbalance arises from the design of YouTube. The platform exposes users to political content, political ads, and a range of possible distractions. If users were offered a meaningful choice to overcome these potential harms, this might already be enough to fix the most acute issues around YouTube. For instance, options might be reducing the personalisation of the user experience by default, reducing the visibility of political content and ads, or democratising some of the decision processes behind YouTube, e. g. through petition platforms to which YouTube must react once a certain threshold of support from users is reached. It should be easy to implement such changes and could bring great benefits to users and society more generally. Yet, any lasting change will require the engagement of all relevant stakeholders. While regulation might be one of many ways to initiate such a process, the will of the users will be essential.

\section{References}

1. "Fiction is outperforming reality": how YouTube's algorithm distorts truth, February 02, 2018. Available at: https://www.theguardian.com/technology/2018/feb/02/how-youtubes-algorithm-distorts-truth (accessed: 13.01.2021).

2. Stein, L. (2013), Policy and Participation on Social Media: The Cases of YouTube, Facebook, and Wikipedia: Policy and Participation on Social Media, Communication, Culture \& Critique, vol. 6, is. 3, pp. 353-371.

3. YouTube's algorithms might radicalise people - but the real problem is we've no idea how they work, January 21, 2020. Available at: http://theconversation.com/youtubes-algorithms-might-radicalise-peoplebut-the-real-problem-is-weve-no-idea-how-they-work-129955 (accessed: 13.01.2021).

4. The trouble with YouTube, August 31, 2006. Available at: https://www.economist.com/business/2006/08/31/the-trouble-with-youtube (accessed: 13.01.2021).

5. YouTube politics, June 25, 2007. Available at: https://www.economist.com/united-states/2007/06/25/ youtube-politics (accessed: 13.01.2021).

6. YouTube launches online EU elections channel, May 6, 2009. Available at: https://www.euractiv.com/ section/languages-culture/news/youtube-launches-online-eu-elections-channel/ (accessed: 13.01.2021).

7. Gillespie, T. (2010), The politics of 'platforms', New Media \& Society, vol. 12, is. 3, pp. 347-364.

8. Ricke, L. (2016), The Impact of Youtube on U.S. Politics. Lanham, MD: Lexington Books.

9. Launching YouTube for Government, October 2, 2014. Available at: https://publicpolicy.googleblog. com/2014/10/launching-youtube-for-government.html (accessed: 13.01.2021).

10. Ridout, T.N. et al. (2015), Politics as Usual? When and Why Traditional Actors Often Dominate YouTube Campaigning, Journal of Information Technology \& Politics, vol. 12, is. 3, pp. 237-251.

11. Helberger, N. (2020), How Current Attempts to Regulate Misinformation Amplify Opinion Power, Digital Journalism, vol. 8, is. 6, pp. 842-854.

12. How YouTube supports elections, February 03, 2020. Available at: https://blog.youtube/news-andevents/how-youtube-supports-elections/ (accessed: 13.01.2021).

13. Are Facebook and YouTube quasi-governmental actors?, August 7, 2018. Available at: https://www. economist.com/democracy-in-america/2018/08/07/are-facebook-and-youtube-quasi-governmental-actors (accessed: 13.01.2021). 
14. Scheufele, D. A. (1999), Framing as a theory of media effects, Journal of Communication, vol. 49, is. 1, pp. 103-122.

15. Pan, Z. and Kosicki, G. M. (1997), Priming and Media Impact on the Evaluations of the President's Performance, Communication Research, vol. 24, is. 1, pp. 3-30.

16. Brewer, P. R., Graf, J. and Willnat, L. (2003), Priming or Framing: Media Influence on Attitudes Toward Foreign Countries, Gazette (Leiden, Netherlands), no. 65 (6), pp. 493-508.

17. Scheufele, D. A. and Tewksbury, D. (2007), Framing, Agenda Setting, and Priming: The Evolution of Three Media Effects Models, Journal of Communication, vol. 57, is. 1, pp. 9-20.

18. Entman, R. M. (2007), Framing Bias: Media in the Distribution of Power, Journal of Communication, vol. 57 , is. 1 , pp. $163-173$.

19. Fairclough, N. (1995), Media discourse. London: Arnold.

20. Eilders, C. (2000), Media as political actors? Issue focusing and selective emphasis in the German quality press, German Politics, vol. 9, is. 3, pp. 181-206.

21. Klinger, U. and Svensson, J. (2015), Network Media Logic: Some Conceptual Considerations, in Bruns, A.et al. (eds.), The Routledge Companion to Social Media and Politics. New York, NY: Routledge, pp. 23-38.

22. Enli, G. S. and Skogerbø, E. (2013), Personalized Campaigns in Party-Centred Politics. Information, Communication \& Society, vol. 16, is. 5, pp. 757-774.

23. Klinger, U. (2013), Mastering the Art of Social Media, Information, Communication \& Society, vol. 16 , is. 5 , pp. $717-736$.

24. Shirky, C. (2011), The Political Power of Social Media: Technology, the Public Sphere, and Political Change, Foreign Affairs, vol. 90, is. 1, pp. 28-41.

25. Page, B. I. (1996), The Mass Media as Political Actors, PS: Political Science and Politics, vol. 29, is. 1, pp. 20-24.

26. Entman, R. M. (1993), Framing: Toward Clarification of a Fractured Paradigm, Journal of Communication, vol. 43 , is. 4 , pp. $51-58$.

27. Olson, M. (2003), The Logic of Collective Action: Public Goods and the Theory of Groups, 21. printing. Cambridge, Mass.: Harvard University Press.

28. Gilens, M. and Page, B. I. (2014), Testing Theories of American Politics: Elites, Interest Groups, and Average Citizens, Perspectives on Politics, vol. 12, is. 3, pp. 564-581.

29. My final letter in 2019: Updates for this year, November 21, 2019. Available at: https://blog.youtube/ inside-youtube/my-final-letter-in-2019/ (accessed: 13.01.2021).

30. A final update on our priorities for 2018, October 22, 2018. Available at: https://blog.youtube/insideyoutube/a-final-update-on-our-priorities-for/ (accessed: 13.01.2021).

31. A bitter fight between YouTube and the EU could have major implications for US tech regulation, December 27, 2018. Available at: https://www.businessinsider.com/youtube-battle-with-the-eu-could-havemajor-implications-for-us-tech-regulation-2018-12 (accessed: 13.01.2021).

32. How YouTube makes users lobby in-house against copyright directive, December 7, 2018. Available at: https://www.euractiv.com/section/digital/news/how-youtube-makes-users-lobby-in-house-against-copyright-directive/ (accessed: 13.01.2021).

33. How Political Ads and Video Content Influence Voter Opinion, March 2016. Available at: https://www. thinkwithgoogle.com/marketing-strategies/video/political-ads-video-content-influence-voter-opinion/ (accessed: 13.01.2021).

34. Dobber, T., Fathaigh, R.Ó. and Borgesius, F.J.Z. (2019), The regulation of online political microtargeting in Europe, Internet Policy Review, vol. 8, is. 4, https://doi.org/10.14763/2019.4.1440

35. YouTube's advertising policy, June 13, 2017. Available at: https://www.europarl.europa.eu/doceo/ document/E-8-2017-003926_EN.html (accessed: 13.01.2021).

36. Answer to Question No E-003926/17, September 15, 2017. Available at: https://www.europarl.europa. eu/doceo/document/E-8-2017-003926-ASW_EN.html (accessed: 13.01.2021).

37. YouTube, the Great Radicalize,. March 10, 2018. Available at: https://www.nytimes.com/2018/03/10/ opinion/sunday/youtube-politics-radical.html (accessed: 13.01.2021).

38. Lewis, R. (2018), Broadcasting the Reactionary Right on YouTube. Data \& Society Research Institute. Available at: https://datasociety.net/wp-content/uploads/2018/09/DS_Alternative_Influence.pdf (accessed: 18.01.2021).

39. Abul-Fottouh, D., Song, M.Y. and Gruzd, A. (2020), Examining algorithmic biases in YouTube's recommendations of vaccine videos, International Journal of Medical Informatics, vol. 140, https://doi. org/10.1016/j.ijmedinf.2020.104175

40. About YouTube. Available at: https://www.youtube.com/intl/en-GB/about/ (accessed: 13.01.2021). 
41. Google to hire thousands of moderators after outcry over YouTube abuse videos, December 5, 2017. Available at: http://www.theguardian.com/technology/2017/dec/04/google-youtube-hire-moderatorschild-abuse-videos (accessed: 13.01.2021).

42. Social media's struggle with self-censorship, October 22, 2020. Available at: https://www.economist. com/briefing/2020/10/22/social-medias-struggle-with-self-censorship (accessed: 13.01.2021).

43. YouTube expands fact-checking feature for video searches to Europe, September 24, 2020. Available at: https://www.cnbc.com/2020/09/24/youtube-expands-fact-checking-feature-for-video-searches-to-europe. html (accessed: 13.01.2021).

44. French blogger claims YouTube tried to censor Juncker interview, September 19, 2016. Available at: http://www.theguardian.com/technology/2016/sep/19/french-blogger-claims-youtube-tried-to-censorjuncker-interview (accessed: 13.01.2021).

45. Press release on YouTube removing the accounts of Krym 24 TV Channel and Anna News and NewsFront news agencies, May 20, 2020. Available at: https://www.mid.ru/maps/us/-/asset_publisher/unVXBbj4Z6e8/content/id/4133893 (accessed: 13.01.2021).

46. Kolotaev, Y. and Kollnig, K. (2020), Perceptions of YouTube’s political influence. Available at: https:// arxiv.org/abs/2012.07745 (accessed: 19.01.2021).

47. Kolotaev, Y. and Kollnig, K. (2019), YouTube: A New Age For Political Campaigning?, The Oxford Student. Available at: https://www.oxfordstudent.com/2019/10/29/youtube-a-new-age-for-political-campaigning/ (accessed: 19.01.2021).

48. I warned in 2018 YouTube was fueling far-right extremism. Here's what the platform should be doing. December 11, 2020. Available at: http://www.theguardian.com/technology/2020/dec/11/youtube-islamophobia-christchurch-shooter-hate-speech (accessed: 13.01.2021).

49. Lynskey, O. (2019), Grappling with "Data Power": Normative Nudges from Data Protection and Privacy, Theoretical Inquiries in Law, vol. 20, is. 1, pp. 189-220.

50. Binns, R. and Bietti, E. (2020), Dissolving privacy, one merger at a time: Competition, data and third party tracking, Computer Law \& Security Review, vol. 36, https://doi.org/10.1016/j.clsr.2019.105369

51. Competition and Markets Authority. Online platforms and digital advertising: Market study final report, July 1, 2020. Available at: https://assets.publishing.service.gov.uk/media/5efc57ed3a6f4023d242ed56/ Final_report_1_July_2020_.pdf (accessed: 18.01.2021).

Received: February 17, 2021

Accepted: March 15, 2021

Authors'information:

Yury Y. Kolotaev - Postgraduate Student; yury.kolotaev@mail.ru

Konrad Kollnig — Postgraduate Student; konrad.kollnig@cs.ox.ac.uk 